\title{
The private value single item bisection auction
}

Citation for published version (APA):

Grigorieva, E., Herings, P. J. J., Vermeulen, A. J., \& Müller, R. J. (2002). The private value single item bisection auction. METEOR, Maastricht University School of Business and Economics. METEOR Research Memorandum No. 035 https://doi.org/10.26481/umamet.2002035

Document status and date:

Published: 01/01/2002

DOI:

10.26481/umamet.2002035

Document Version:

Publisher's PDF, also known as Version of record

\section{Please check the document version of this publication:}

- A submitted manuscript is the version of the article upon submission and before peer-review. There can be important differences between the submitted version and the official published version of record.

People interested in the research are advised to contact the author for the final version of the publication, or visit the DOI to the publisher's website.

- The final author version and the galley proof are versions of the publication after peer review.

- The final published version features the final layout of the paper including the volume, issue and page numbers.

Link to publication

\footnotetext{
General rights rights.

- You may freely distribute the URL identifying the publication in the public portal. please follow below link for the End User Agreement:

www.umlib.nl/taverne-license

Take down policy

If you believe that this document breaches copyright please contact us at:

repository@maastrichtuniversity.nl

providing details and we will investigate your claim.
}

Copyright and moral rights for the publications made accessible in the public portal are retained by the authors and/or other copyright owners and it is a condition of accessing publications that users recognise and abide by the legal requirements associated with these

- Users may download and print one copy of any publication from the public portal for the purpose of private study or research.

- You may not further distribute the material or use it for any profit-making activity or commercial gain

If the publication is distributed under the terms of Article $25 \mathrm{fa}$ of the Dutch Copyright Act, indicated by the "Taverne" license above, 


\title{
The private value single item bisection auction
}

\author{
Elena Grigorieva * P.Jean-Jacques Herings ${ }^{\dagger} \quad$ Rudolf Müller ${ }^{\ddagger}$ \\ Dries Vermeulen $\S$
}

November 15, 2002

\begin{abstract}
In this paper we present a new iterative auction, the bisection auction, that can be used for the sale of a single indivisible object. We will show that the bisection auction is computationally more efficient than the classical English auction while it still preserves all characteristics the English auction shares with the Vickrey auction: there exists an equilibrium in weakly dominant strategies in which everyone truthfully reveals his information, the object is allocated in accordance with efficiency requirements to the buyer who has the highest valuation, and the price paid by the winner of the object equals the second-highest valuation.
\end{abstract}

JEL Codes. C72, D44.

Keywords. Single item auction; Nash equilibria; weakly dominant strategy, extensive form games of incomplete information.

\section{Introduction}

An auction is a mechanism that can be used to sell goods to potential buyers. In this paper we will only be concerned with the sale of a single indivisible object, for example a collectible such as a stamp or a painting. It is supposed that each of the potential buyers has a certain valuation for the object. A buyer knows exactly what the object is worth to him. But he does not know the precise valuations of the other potential buyers for the object, neither does the seller. In this setting an auction is a very powerful tool for selling the object, for many different reasons ([8], [10], [11]).

An auction format has three ingredients.

\footnotetext{
${ }^{*}$ e.grigorieva@ke.unimaas.nl. Department of Quantitative Economics, Universiteit Maastricht, P.O. Box 616, 6200 MD Maastricht, The Netherlands. The author acknowledges support by the Dutch Science Foundation NWO through grant 401-01-101.

${ }^{\dagger}$ p.herings@algec.unimaas.nl. Department of Economics, Universiteit Maastricht, P.O. Box 616, 6200 MD Maastricht, The Netherlands.

${ }^{\ddagger}$ r.muller@ke.unimaas.nl. Department of Quantitative Economics, Universiteit Maastricht, P.O. Box 616, 6200 MD Maastricht, The Netherlands. The author acknowledges support by European Commission through funds for the International Institute of Infonomics.

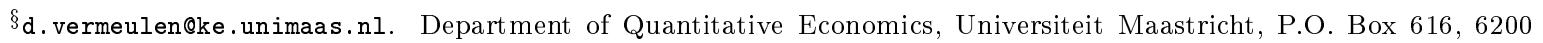
MD Maastricht, The Netherlands.
} 
(1) The auction format specifies which bids are allowed to be made. In other words, this part of the format describes the strategic possibilities available to the buyers. There is much choice on how to design this part of the auction and bids can take many different forms.

(2) Given the bids (or strategies) of the buyers, the auction format provides an algorithm to decide who gets the object. In other words, in this part of the auction it is specified how to compute the winner of the auction.

(3) Given the bids, the auction also provides an algorithm that computes the payments to be made from the buyers to the auctioneer. Although usually only the winner is supposed to pay, this need not always be the case.

One of the best known classical auction formats is the English auction. Traditionally the English auction is used to sell one single indivisible object. Typically, in the English auction a group of potential buyers gathers in a room and consecutively announce their bids, a bid being the price the bidder in question is willing to pay for the object at that moment. So, basically anyone is allowed to make any bid at any moment. The only rule, apart from a couple of very obvious ones such as "you are not allowed to withdraw a bid" and "buyers are not allowed to collude", is that each bid should be higher than the previous one. The last buyer to make a bid is declared the winner of the auction. He gets the object and the price he pays is equal to the final bid he made.

In his famous 1961 contribution to auction theory, Vickrey [16] showed that the sealed-bid secondprice auction format (for obvious reasons also known nowadays as the Vickrey auction) is strategically equivalent to the English auction ${ }^{1}$. Under this auction format bidders are only allowed to submit one single (sealed) bid. The buyer with the highest bid is declared to be the winner. He gets the object for a price equal to the second-highest bid that is made. Using this one-shot version of the English auction, Vickrey established two main results. First of all he showed that truth-telling -that is, bidding your true valuation for the object- is a weakly dominant strategy, independent of the a priori distribution of the valuations of the buyers. Secondly, he observed that the resulting truth-telling equilibrium is indeed efficient (Pareto optimal) in the sense that the object gets assigned to the buyer who values it most.

Yet both the English auction and the Vickrey auction do have their drawbacks in practical use. In terms of applicability the Vickrey auction is probably the worst performer. Although the principle of the Vickrey auction is very easy to understand and the execution of the auction can be done very fast (it lasts exactly one round) most people are somewhat troubled when they are asked to participate in a

\footnotetext{
${ }^{1}$ Well, not exactly. In the original English auction the highest price is charged. This, as Vickrey already observed, only approximates the Vickrey auction, and truth-telling is not a dominant strategy. However, if also in the iterative auction one still charges the second price, all features of the one-shot version remain intact!
} 
Vickrey auction pur sang, especially when the stakes are high. One possible reason for this is that the Vickrey auction format requires all participants to reveal all information they have [14]. Besides that, even though valuations are private, they might be correlated. And since in reality people are not always sure about their own valuations, partial information on other people's value for the object helps people to estimate their own valuations [1], [9]. Thus, in such situations, an iterative auction, e.g. English auction is generally preferable on information-revelation grounds [4]. Sealed bidding denies bidders the opportunity to learn about other people's information during the course of the auction, and experiments demonstrate that ascending auctions perform better than Vickrey auctions, because the incentive for playing the efficient dominant strategy is clearer to bidders [7].

The English auction has its distinct practical disadvantage. A significant problem with such an auction format is that it might proceed at a very slow pace. E.g., the bidders might choose the increment with which the price goes up too small, in which case the auction might take a long and unpredictable amount of time to reach the required price level. One remedy is to introduce a minimum or a fixed bid increment, as has been done for example in the spectrum auctions. This though is known to affect the efficiency of the auction [15]. In [3] Chwe presents a model of the economic trade-off between auction duration and step size for oral auctions where bids are restricted to discrete levels. Recognition of the speed problem raises the question of how to design an efficient iterative auction that is fast. We are aware of only a single paper that deals with issues related to the design of new fast auctions. In [5] the authors propose a family of hybrid auctions, called "survival auctions" which combine the benefit of both sealed-bid auctions (quick with predictable termination time) and ascending-bid auctions (they possess an information-revelation component).

The design of the auction defines not only the rules by which the auctioneer decides on the allocation and price, but also the rules by which bidders report their bids and the rules saying which information is given from the auctioneer to the bidders. This together defines the communication complexity of the auction. Various communication considerations on the design of auctions were pointed out by several researchers. Recognition of the communication problem has prompted researchers to examine the properties of auctions with different types of information exchange between participants. For the most simple case of selling a single indivisible object [13] studies the effect of severely restricting the amount of communication allowed in an auction. In [2] Ausubel and Milgrom examine the behavior of ascending-bid multi-item auctions with restricted communication possibilities. In [12] Nisan et al. analyze the minimum amount of communication required to achieve or approximate allocation efficiency. 
The primal contribution of this paper is to present and to analyze an alternative iterative auction format, called the "bisection auction", that is computationally fast as well as economical in its demand for information, and that has the same performance characteristics as the Vickrey auction by mimicking a second price auction. In particular, it preserves efficiency and truth telling remains a dominant strategy. The underlying principle of this auction is based on the bisection method, a well-known fast algorithm that can be used e.g. for the determination of realizations of random variables on a given interval.

Suppose a single indivisible object is auctioned. We assume that buyers are risk neutral, their valuations are supposed to be integer, randomly drawn from a bounded interval - by default of the form $\left[0,2^{R}\right)$ for some positive integer $R$. The bisection auction has $R$ rounds. The price sequence starts at the middle of the initial interval with a price equal to $2^{R-1}$. Bidders report their demand at the current price by sealed bids. A yes-bid stands for the announcement to be willing to buy at the current price, a no-bid for the contrary. As a function of these bids, the auctioneer announces the price of the next round.

In case there are at least two players submitting a yes-bid, the price goes up to the middle of the upper half interval, i.e. to the interval $\left[2^{R-1}, 2^{R}\right)$. The buyers that are allowed to participate actively in the next round are the ones that said yes and they are competing for the object in the price range $\left[2^{R-1}, 2^{R}\right)$. The other players drop out of the auction, and do no longer have any influence on the proceedings of the auction. In case there is at most one player saying yes, attention shifts to the lower half interval and the price goes down. Two different things can happen now. First, the easy case, if no-one submits a yes-bid. In that case all active buyers remain active in the next round, the price goes down to the middle of the lower half interval, i.e. the interval $\left[0,2^{R-1}\right)$. In the other case there is a single buyer that submitted a yes-bid. This buyer now becomes the winner and he gets the object. Nevertheless (remember that we are mimicking a second-price auction) the auction doesn't end, but enters a price-determination phase. Once we enter this price-determination phase, the price goes down, and the active players in the next round are the ones that were active in the previous round minus the winner. They are competing on the lower half interval $\left[0,2^{R-1}\right){ }^{2}$ The winner, although he is no longer considered to be active, is considered to say yes to all prices that are proposed beyond the moment he became the winner. After all, all these prices will be lower than the price he agreed to when he became a winner. Apart from this, the way it is decided whether the price should go up or down is not any different from the way this is decided in the winner-determination phase. In each round depending on submitted bids we subsequently restrict attention to either the lower half of the current interval, or to the upper half of the current interval.

\footnotetext{
${ }^{2}$ Concerning the information policy it is important that bidders cannot observe all bids of the other bidders. In order to keep active buyers motivated to participate in the auction they do not get to know that the object has already been assigned.
} 
Iterating this procedure will eventually yield a winner ${ }^{3}$ and a price. The price is uniquely determined because in each round the length of the current interval goes down by half. Since the initial interval is of length $2^{R}$, after $R$ rounds the resulting interval is of length 1 . And since it is a half-open interval, it contains exactly one integer. This integer is declared to be the price the winner of the auction has to pay for the object.

The paper is organized as follows. In Section 2 we introduce a formal model of the bisection auction and derive a few basic results on the internal logic of the construction we present. In Section 3 we focus on the strategic analysis of the auction. We show that, despite appearances, a player in the auction game only has a couple of fairly straightforward strategies, so-called threshold strategies. Such a strategy simply states that the bid is yes if the price mentioned by the auctioneer is lower than the threshold in case, otherwise it is no. Using this result we subsequently show that the buyer with the highest threshold gets the object and the price the winner pays is equal to the second-highest threshold. Interpreting the thresholds as bids in the Vickrey auction we consequently get the same outcome in the bisection auction as in the Vickrey auction. Thus, we show that the bisection auction is strategically equivalent to the Vickrey auction (and hence also to the English auction). Strategic equivalence of these auctions implies that, as long as bidders behave rationally, in all these auctions the same bidder will win and pay the same amount. Thus, we claim that like in the Vickrey auction, also in this auction truth-telling - that is, choosing the threshold equal to your valuation - is a weakly dominant strategy and the equilibrium results in an efficient allocation. We conclude with a few comments about the relevance of our results, comparing in Section 4 the speed and informational requirements of the English, the Vickrey and the bisection auctions. We claim that the proposed auction is preferred over the Vickrey auction because of its iterative nature and over the English auction because of its speed. In the bisection auction the query of announced prices converges to the smallest Walrasian price in logarithmic time. This guarantees a fast and predictable termination of the auction, while the running time of the English auction is of exponential size relative to the length of the binary representation of the valuations of the buyers.

\section{The bisection auction}

In the introduction we already gave an intuitive description of the bisection auction. Our purpose though, which is to obtain a formal proof that truth-telling is a dominant strategy in this setting, requires a more exact framework. For this reason we will in this section represent the bisection auction by an extensive form game with imperfect information.

\footnotetext{
${ }^{3}$ Unless in no round there is precisely one player that said yes. In that case several players will still be active after $R$ rounds, and the object is assigned by a lottery.
} 
Formally we would need to introduce a move of nature that represents the realization of the random variable of a player's valuation. But to simplify the analysis we deviate somewhat from the classical approach to games of this sort. We will not include a move of nature in the tree to represent the realization of the valuations but rather construct the tree independent of these realizations. In fact, what we will construct can be interpreted as a game board on which the auction game can be played, together with a booklet (basically Section 2.1) in which the rules of the game are explained. Any group of players, whose valuations for the object to be sold are not known to the designer of the game, can buy this package (the game board and the booklet) and play the game if they wish to do so.

The game board itself is constructed as follows. Since players are allowed to let the decisions they take during the game depend on the information they have concerning past actions of themselves and others, the game board is simply the collection of all recordings of sequences of actions (histories). Further, since for each such sequence the actions a player can take are saying yes or no, the result is a binary tree.

Next, notice that the rules of the auction require players to move simultaneously in each round. However, since we model the auction in an extensive form setting, this is not explicitly possible, but takes the form of sequential moves in which the players don't observe moves made by the other players. Thus, one more ingredient of the game board is a description of how histories are grouped together into information sets. The information sets reflect the fact that there is no full disclosure, and that the players only get partial information on the proceedings of the game.

\subsection{Formal representation of the auction}

Now we will be a bit more precise and describe the game in full detail. First we introduce some elementary data.

(1) A finite set of players $N=\{1, \ldots, n\}$ that participate in the game.

(2) A number of rounds $R$ that specifies the duration of the game.

(3) For each player a decision set $A=\{y e s, n o\}$. This reflects the fact that each player has to make a binary decision in each and every of his information sets.

Given these basic data we can specify the "game board" which takes the form of a binary tree. We will specify both the nodes and the edges of the tree formally.

(4) Every node in the game tree is a vector $a$ that represents the history of decisions chosen by players before the game reached this node. Formally, $a=\left(a_{k}\right)_{k=1}^{r}$ with $1 \leq r \leq R$ where $a_{k}=\left(a_{k i}\right)_{i=1}^{n}$ for $k<r$ 
and $a_{r}=\left(a_{r i}\right)_{i=1}^{j}$ for some $j \leq n$. Here $a_{k i}$ is a particular decision in the set $A$ chosen by player $i$ in round $k$ (so it is either a yes or a no).

We will need the following notational conventions in this context. The length of a node is defined as $l(a)=(r-1) n+j$. The initial node $a_{0}$ is a node with length equal to 0 . This node corresponds to the first round where the first player has to make a decision. The nodes with length equal to $r n$ are referred to as the start of round $r+1$. The terminal nodes are nodes whose length is equal to $R n$. These nodes correspond to the end of the game.

Switching back to the description of the game board we will now specify the edges of the binary tree.

(5) There is an edge between two nodes $a$ and $b$ if the length of $b$ is equal to the length of $a$ plus one, and for all $i$ and $k$ for which $a_{k i}$ is defined, $a_{k i}=b_{k i}$. This is the obvious way to define edges once you recall that the nodes defined above represent recorded histories of past actions taken by the players. So, there is an edge between two nodes if in the second node one player has made an additional decision in comparison with the first node.

The next ingredient of the description of the game board is the specification of the information sets in a given round $r$. Let $X_{r}$ denote the set of nodes corresponding to round $r$. This set $X_{r}$ partitions into $n$ sets $X_{r i}$, each $X_{r i}$ being the set of nodes in round $r$ where player $i$ has to make a decision. Formally, $X_{r i}=\{a \mid l(a)=(r-1) n+i-1\}$.

(6) Every set $X_{r i}$ splits into several information sets. In order to define those, and also to highlight the logic of the auction we define here, we associate with every node $a$ three sets of players.

$A(a)$ - the set of players that are active in node $a$

$W(a)$ - the winner set in node $a$ (this set will consist of at most one player)

$D(a)$ - the set of players that dropped out before the game reached node $a$.

These three sets partition the player set $N$. Furthermore, we introduce one more additional set. This set is not fundamental, but it does help to understand the logic of the auction game we are constructing. $P(a)$ - the set of players whose decisions made in the last completed round (before the game reaches node a) are counted as yes. This set consists of those active players whose decision in the previous round was yes together with the winner in the previous round (if there was one).

As we will see in the next section, in each round the players get to hear whether this particular set $P(a)$ does or does not have more than one member. This information and the players' own previous actions identify the players' information sets. 
Now we turn to the formal definition of these sets. For the initial node $a_{0}$ they are defined as follows: $A\left(a_{0}\right)=N, W\left(a_{0}\right)=\emptyset, D\left(a_{0}\right)=\emptyset$ and $P\left(a_{0}\right)=\emptyset$.

Now we iteratively define them for nodes with arbitrary length. We introduce a notational convention.

For a node $a \in X_{r}$ and $q<r$, let $a^{q}=\left(a_{k}\right)_{k=1}^{q}$ denote the part of the history $a$ corresponding to the first $q$ rounds. Now, for a history $a=\left(a_{k}\right)_{k=1}^{r}$ with $l(a)=n r$, the sets $A(a), W(a), D(a)$, and $P(a)$ are defined as follows.

$$
\begin{aligned}
& P(a)=W\left(a^{r-1}\right) \cup\left\{i \in A\left(a^{r-1}\right) \mid a_{r i}=y e s\right\} \\
& W(a)=\left\{\begin{array}{lll}
W\left(a^{r-1}\right) & \text { if } \quad W\left(a^{r-1}\right) \neq \emptyset \\
\emptyset & \text { if } & W\left(a^{r-1}\right)=\emptyset \text { and }|P(a)| \neq 1 \\
P(a) & \text { if } & W\left(a^{r-1}\right)=\emptyset \text { and }|P(a)|=1
\end{array}\right. \\
& D(a)= \begin{cases}D\left(a^{r-1}\right) & \text { if }|P(a)| \leq 1 \\
D\left(a^{r-1}\right) \cup\left\{i \in A\left(a^{r-1}\right) \mid a_{r i}=n o\right\} & \text { otherwise }\end{cases} \\
& A(a)=N \backslash(W(a) \cup D(a)) .
\end{aligned}
$$

For a history $a=\left(a_{k}\right)_{k=1}^{r}$ with $l(a) \neq n r$, we define these sets as follows: $P(a)=P\left(a^{r-1}\right), W(a)=$ $W\left(a^{r-1}\right), D(a)=D\left(a^{r-1}\right)$ and $A(a)=A\left(a^{r-1}\right)$.

Remarks. For all $a \in X_{1}$ we have $A(a)=N, W(a)=D(a)=P(a)=\emptyset$. Furthermore, for any two nodes $a$ and $b$ in $X_{r}$ with $a^{r-1}=b^{r-1}$ we have equality of the sets defined above, that is $P(a)=P(b)$, $W(a)=W(b), D(a)=D(b)$ and $A(a)=A(b)$.

After this preliminary work we can define the information sets themselves. We basically do this by means of an equivalence relation.

Definition 2.1 Two nodes $a$ and $b$ from $X_{r i}$ are said to be in the same information set if for all $k<r$

1. $a_{k i}=b_{k i}$

2. $\left|P\left(a^{k}\right)\right| \leq 1$ iff $\left|P\left(b^{k}\right)\right| \leq 1$.

Notice that this is a sensible definition since both 1 and 2 are equivalence relations. Thus the information sets are simply the equivalence classes corresponding to these relations. We will denote the information set containing node $a$ by $H(a)$. Furthermore, an information set $H(a) \subset X_{r i}$ is said to represent a sufficient vote if $\left|P\left(a^{r-1}\right)\right|>1$ and an insufficient vote otherwise. 
(7) The vector $p(\tau)=\left(p_{i}(\tau)\right)_{i=1}^{n}$ of payments for each terminal node $\tau$. To define this vector we associate with each terminal node $\tau$, in addition to the sets mentioned above, a set $W I N(\tau)$ that is a set of candidate winner(s) of the game if the game terminates in node $\tau$. This set is defined by

$$
W I N(\tau)= \begin{cases}W(\tau) & \text { if } W(\tau) \neq \emptyset \\ A(\tau) & \text { otherwise. }\end{cases}
$$

If $|W I N(\tau)|>1$, then the winner of the game is determined by a lottery among all players $i$ in $W I N(\tau)$, otherwise it is the buyer in $W(\tau)$. The price winner $j$ pays is

$$
p_{j}(\tau)=2^{R-1}+\sum_{k=1}^{R-1} \lambda_{k} 2^{R-k-1}+\beta
$$

where for all $k$

$$
\lambda_{k}=\left\{\begin{aligned}
-1 & \text { if }\left|P\left(\tau^{k}\right)\right| \leq 1 \\
1 & \text { otherwise }
\end{aligned} \quad \text { and } \quad \beta=\left\{\begin{aligned}
-1 & \text { if }|P(\tau)| \leq 1 \\
0 & \text { otherwise. }
\end{aligned}\right.\right.
$$

All other players pay zero, so $p_{i}(\tau)=0$ for all $i \neq j$.

A few remarks. In the same way we can associate a price with an arbitrary internal node. This works as follows. For a node $a=\left(a_{k}\right)_{k=1}^{r}$ with $l(a)=n r$ we define its price by

$$
p(a)=2^{R-1}+\sum_{k=1}^{r} \lambda_{k} 2^{R-k-1}
$$

where for all $k$

$$
\lambda_{k}=\left\{\begin{aligned}
-1 & \text { if }\left|P\left(a^{k}\right)\right| \leq 1 \\
1 & \text { otherwise. }
\end{aligned}\right.
$$

Note that for $a$ with $l(a)=n r$ the price $p(a)=p\left(a^{r-1}\right)+2^{R-r-1}$ if $|P(a)|>1$ and $p(a)=p\left(a^{r-1}\right)-$ $2^{R-r-1}$ if $|P(a)| \leq 1$. Furthermore, for a node $a=\left(a_{k}\right)_{k=1}^{r}$ with $l(a) \neq n r$ we define $p(a)=p\left(a^{r-1}\right)$.

These internal prices are very much intertwined with the logic of the auction. In any node $a$ we now have a price. And the actions a player can take in this node $a$, either a yes or a no, can be interpreted as being his answer to the question:

Are you willing to pay this (current) price $p(a)$ for the object we are selling?

Moreover, these prices can be used to characterize information sets. Namely, the second requirement in the condition for nodes to be in one information set is satisfied if and only if these nodes are labelled by the same price. So we can conclude that for any $a, b \in X_{r i}$ from the same information set, $p(a)=p(b)$ while two different information sets of the same player in the same round will indeed have different prices (or a different choice by the player himself in a previous round). Thus, each information set in $X_{r i}$ can be represented by its associated price and the sequence of decisions chosen by player $i$ in the first $r-1$ rounds. 


\subsection{An exploration of the game board}

So far we only concentrated on the construction of what we called the "game board". Now we will develop some theory that provides a little more insight in how the game looks like. We will need this insight in the next section, where we will characterize optimal playing behavior for the players of the auction game.

The first, still rather abstract, observation we need to make is the following lemma. Roughly speaking it states the following. Suppose that we have a history $a$ and further suppose that each player takes the same actions in another history $b$ as long as he is an active player according to $a$. Then, every player stayed active in $b$ precisely until the moment he also became non-active in $a$, no matter what any players have done in $b$ when they were no longer active. Moreover, all other sets defined above depend only on the actions of active players, too. Formally,

Lemma 2.2 Suppose we have a history $a=\left(a_{k}\right)_{k=1}^{r}$. Further, suppose that $b$ is a history such that $l(b)=l(a)$ and $b_{k i}=a_{k i}$ for all $i \in A\left(a^{k-1}\right)$ for all $k \leq r$. Then $P(a)=P(b), W(a)=W(b)$, $D(a)=D(b)$, and $A(a)=A(b)$.

Proof. We will actually show that we have equality of all these sets for all histories $a^{k}$ and $b^{k}$ for all $k \leq r$. We will show this inductively for each round $k$.

1. For $k=1$. Since in the first round all players are active, we have $a^{1}=b^{1}$. Hence, it follows immediately that $P\left(a^{1}\right)=P\left(b^{1}\right), W\left(a^{1}\right)=W\left(b^{1}\right), D\left(a^{1}\right)=D\left(b^{1}\right)$, and $A\left(a^{1}\right)=A\left(b^{1}\right)$.

2. Suppose the lemma is true for some $k$. We have to show that the lemma must also be true for $k+1$. Since $A\left(a^{k}\right)=A\left(b^{k}\right)$ and by assumption it holds that $a_{k+1, i}=b_{k+1, i}$ for all players $i$ in $A\left(a^{k}\right)$, we can conclude that

$$
\left\{i \in A\left(a^{k}\right) \mid a_{k+1, i}=y e s\right\}=\left\{i \in A\left(b^{k}\right) \mid b_{k+1, i}=y e s\right\} .
$$

Together with the fact that $W\left(a^{k}\right)=W\left(b^{k}\right)$ this implies $P\left(a^{k+1}\right)=P\left(b^{k+1}\right)$. Once we know that, the fact that $W\left(a^{k}\right)=W\left(b^{k}\right)$ also implies that $W\left(a^{k+1}\right)=W\left(b^{k+1}\right)$. Of course we also know that

$$
\left\{i \in A\left(a^{k}\right) \mid a_{k+1, i}=n o\right\}=\left\{i \in A\left(b^{k}\right) \mid b_{k+1, i}=n o\right\} .
$$

Together with $D\left(a^{k}\right)=D\left(b^{k}\right)$ and $P\left(a^{k+1}\right)=P\left(b^{k+1}\right)$ this implies $D\left(a^{k+1}\right)=D\left(b^{k+1}\right)$. Finally, since $W\left(a^{k+1}\right)=W\left(b^{k+1}\right)$ and $D\left(a^{k+1}\right)=D\left(b^{k+1}\right)$, we have $A\left(a^{k+1}\right)=A\left(b^{k+1}\right)$.

Notice that since the actions of all active players are the same, the fact that $P\left(a^{k}\right)=P\left(b^{k}\right)$ for all $k<r$ implies that as long as a player is active, he reaches the same information sets in both histories. 
Moreover, as long as a nonactive player has the same sequence of own decisions he reaches the same information sets in both histories. Hence,

Corollary 2.3 For two histories a and b like in lemma 2.2 any player that takes the same decisions in both histories reaches the same information sets in all rounds.

At this point we would like to make one more straightforward observation. Suppose we have a player $i$ who happens to be active in a given information set. Further suppose that in this information set he takes the decision yes. Then there is only one subsequent (that is, immediate successor) information set in which this player is still active, namely the one in which there is a sufficient vote.

This observation is an immediate consequence of the definition of an information set. Once the player in question decides to play yes, there are two possible information sets he can find himself in in the next round. One in which there is an insufficient vote, in which case he became winner (and therefore no longer active) and one in which there is a sufficient vote, in which case he is still active. Of course, a similar remark can be made concerning an active player that decides to play no. All in all, this shows the following proposition.

Proposition 2.4 For an information set in which player $i$ is active and for every decision of player $i$ made in this set, there exists exactly one immediate successor information set in which player $i$ is still active. Moreover, this immediate successor is, in case the player chose yes, the one with a sufficient vote, and the one with an insufficient vote in the other case.

\section{Playing the game}

So far we only considered what we earlier described as the "game board" and the "booklet" of game rules. We did not yet discuss the people who are supposed to "play the game", that is, the buyers who participate in the auction. Those are our concern in this section. We will briefly discuss how valuations and payoffs for these buyers are defined and then analyze the strategic possibilities of the players. We will show that, despite first appearances when we think in terms of information sets, the players do not have much freedom of choice. Essentially each strategy can be characterized by one single real number, which we call the threshold. Using this result we will a.o. show that usage of a player's valuation as his threshold is a weakly dominant strategy.

We will first specify the payoffs of the players in terminal nodes. Let the valuations of the players be given by $v=\left(v_{i}\right)_{i=1}^{n}$, where $v_{i}$ is the valuation player $i$ assigns to the object. Now let $\tau$ be a terminal 
node. The payoff of player $i$ in terminal node $\tau$ is defined by

$$
U_{i}(\tau)= \begin{cases}\left(v_{i}-p(\tau)\right) & \text { with probability } \frac{1}{|W I N(\tau)|} \text { if } i \in W I N(\tau) \\ 0 & \text { else. }\end{cases}
$$

Notice that we deviate somewhat from the usual way of modeling moves of nature. If there is more than one player in the set $W I N(\tau)$ of candidate winners we do not explicitly model the random draw that determines the winner of the object. We rather simply observe that, according to (7) of the formal definition of the auction game, player $i$ has probability $\frac{1}{|W I N(\tau)|}$ of getting the object if he is a member of $W I N(\tau)$ and will therefore have a payoff of $v_{i}-p(\tau)$ with probability $\frac{1}{|W I N(\tau)|}$. In the remaining cases -so either not being a member of $W I N(\tau)$ at all or being a member of $W I N(\tau)$ but losing the lottery- he does not get assigned the object, pays nothing, and consequently ends up with payoff 0 .

\subsection{Equivalence of strategies and threshold strategies}

Now that we know how the players evaluate the outcomes of the auction we just designed, we can also start to analyze how players could, and should, behave in this auction. Let $\mathcal{H}_{i}$ denote the collection of player $i$ 's information sets.

Definition 3.1 A strategy for player $i$ is a function that assigns to each information set $H \in \mathcal{H}_{i}$ of that player an action $s_{i}(H)$ in $A$.

First notice that the number of information sets of each player corresponding to round $k$ is equal to $4^{k-1}$ (unless the number of players equals two, in which case it is $3^{k-1}$ ). So, the total number of information sets in the game for each player is equal to $\sum_{k=1}^{R} 4^{k-1}=\frac{1}{3}\left(4^{R}-1\right)$. Thus, the number of possible strategies of a player equals $2^{\frac{1}{3}\left(4^{R}-1\right)}$, quite a staggering amount. Nevertheless, we will show that effectively the number of really essentially different strategies is much lower, in the order of $2^{R}$ to be specific.

In order to see this, first observe that the behavior of players will very much depend on the way what we called the internal prices develop. This works as follows. Once we have reached node $a$ in round $r$, the player who has the move at that moment knows that the price will move at most $2^{R-r}-1 .{ }^{4}$ Moreover, if a player says no to that price, he is certain that he will not have to pay more than this internal price in case he wins the object.

We will make clear in a moment that it is not attractive to say no to a certain price, and say yes to a higher price, or to say yes to a certain price, and say no to a lower price. This brings us to the following

\footnotetext{
${ }^{4}$ Notice that the price moves at most with the amount $\sum_{k=r}^{R-1} 2^{R-k-1}$ that is equal to $2^{R-r}-1$.
} 
specific type of strategy for player $i$. Let $H$ be an information set of player $i$ in round $r$ and let $p(H)$ denote the price corresponding to that information set.

Definition 3.2 Let $t$ be an integer. The threshold strategy $s_{i}^{t}$ is the function that assigns the action yes to each information set $H \in \mathcal{H}_{i}$ with $p(H) \leq t$ and the action no to each information set $H \in \mathcal{H}_{i}$ with $p(H)>t$. The number $t$ is called the threshold.

We show next that from a strategic perspective the collection of threshold strategies is sufficient. To be a bit more precise, for any strategy of a player there is a threshold strategy of this player such that, no matter what strategies the other players use, this player will do the same under both strategies as long as he is active. Consequently, no other player can distinguish between these two strategies. The information sets that will be reached by other players as well as the payoffs of all players are the same under both strategies.

In order to derive this result, we first define for each (arbitrary) strategy of a player the threshold of its realization-equivalent threshold strategy. For this we first need some preliminary results on how prices move during the game compared to the actions taken by active players.

Lemma 3.3 Let $s_{i}$ be a strategy of player $i$ and let $r$ be a round. Then there is a unique information set $H_{r, i}\left(s_{i}\right)$ of player $i$ such that he is necessarily in information set $H_{r, i}\left(s_{i}\right)$ if he is still active in round $r$. Furthermore, the price of this information set $H_{r, i}\left(s_{i}\right)$ equals

$$
p\left(H_{r, i}\left(s_{i}\right)\right)=2^{R-1}+\sum_{k=1}^{r-1} \lambda_{k} 2^{R-k-1}
$$

where for all $k$

$$
\lambda_{k}=\left\{\begin{array}{rll}
-1 & \text { if } & s_{i}\left(H_{k, i}\left(s_{i}\right)\right)=\text { no } \\
1 & \text { if } & s_{i}\left(H_{k, i}\left(s_{i}\right)\right)=\text { yes. }
\end{array}\right.
$$

Proof. First for round $r=1$. The information set $H_{1 i}\left(s_{i}\right)$ is simply equal to the unique information set $X_{1 i}$ of player $i$ in round 1 . Its price is $p\left(H_{1 i}\left(s_{i}\right)\right)=2^{R-1}$.

Now suppose that for some $r<R$ the information set $H_{r, i}\left(s_{i}\right)$ has been reached (and its price is determined as above). Since player $i$ is supposed to be active in this information set, according to Proposition 2.4 the action $s_{i}\left(H_{r, i}\left(s_{i}\right)\right)$ player $i$ takes in this information set determines a unique immediate successor $H_{r+1, i}\left(s_{i}\right)$ of $H_{r, i}\left(s_{i}\right)$ in which player $i$ is still active.

Furthermore, for $s_{i}\left(H_{r, i}\left(s_{i}\right)\right)=n o$, this unique information set is reached by an insufficient vote. This implies that the price of $H_{r+1, i}\left(s_{i}\right)$ is equal to $p\left(H_{r, i}\left(s_{i}\right)\right)-2^{R-r-1}$. Thus, $\lambda_{r}=-1$ in this case. If on 
the other hand $s\left(H_{r, i}\left(s_{i}\right)\right)=y e s$, this unique information set is reached by a sufficient vote. This implies that the price of $H_{r+1, i}\left(s_{i}\right)$ is equal to $p\left(H_{r, i}\left(s_{i}\right)\right)+2^{R-r-1}$ and hence $\lambda_{r}=1$.

This enables us to specify for any strategy its equivalent threshold strategy.

Definition 3.4 Let $s_{i}$ be a strategy of player $i$. The threshold $t_{i}\left(s_{i}\right)$ corresponding to $s_{i}$ is defined by

$$
t_{i}\left(s_{i}\right)=p\left(H_{R, i}\left(s_{i}\right)\right)+\beta
$$

where

$$
\beta=\left\{\begin{array}{rll}
-1 & \text { if } & s_{i}\left(H_{R, i}\left(s_{i}\right)\right)=\text { no } \\
0 & \text { if } & s_{i}\left(H_{R, i}\left(s_{i}\right)\right)=\text { yes. }
\end{array}\right.
$$

Now we will show that a strategy $s_{i}$ and the threshold strategy whose threshold is equal to the threshold $t_{i}\left(s_{i}\right)$ corresponding to $s_{i}$ are realization-equivalent. Given the strategies of other players, whether player $i$ chooses strategy $s_{i}$ or threshold strategy $\widetilde{s_{i}}$ with threshold equal to $t_{i}\left(s_{i}\right)$, the realization of the game doesn't change. We will show that, and even a bit more than that, in the next theorem.

Theorem 3.5 Let $s_{i}$ be a strategy and let $\widetilde{s_{i}}$ be the threshold strategy with threshold $t_{i}\left(s_{i}\right)$. Let $s_{-i}$ be an arbitrary collection of strategies for players other than $i$. Let a be the realized history if $s=\left(s_{-i}, s_{i}\right)$ is played and let $b$ be the realized history if $\widetilde{s}=\left(s_{-i}, \widetilde{s_{i}}\right)$ is played. Then for both histories holds 1. For every round $r, A\left(a^{r-1}\right)=A\left(b^{r-1}\right), D\left(a^{r-1}\right)=D\left(b^{r-1}\right)$ and $W\left(a^{r-1}\right)=W\left(b^{r-1}\right)$.

2. For every round $r$ where player $i$ is active according to a we have $a^{r}=b^{r}$.

3. For all $k \leq r$ and all players $j \in A\left(a^{k-1}\right), a_{k j}=b_{k j}$.

4. All players $j \neq i$ in all rounds reach the same information sets.

5. Consequently, all payoffs are the same.

Proof. Using Lemma 3.2 we will first prove statements 1, 2 and 3 by induction.

(I) For round $r=1$. By definition, $A\left(a^{0}\right)=A\left(b^{0}\right), D\left(a^{0}\right)=D\left(b^{0}\right)$ and $W\left(a^{0}\right)=W\left(b^{0}\right)$. So, (1) is true in this case.

In order to prove (2) and (3), notice that for any player $j$ the set $X_{1 j}$ is the only information set of this player in round 1. Furthermore, since each player $j$ is active in round 1, we need to show that

$$
s_{j}\left(X_{1 j}\right)=\widetilde{s}_{j}\left(X_{1 j}\right)
$$

for every player $j$. Since $s_{j}=\widetilde{s}_{j}$ for all $j \neq i$ it suffices to prove that $s_{i}\left(X_{1 i}\right)=\widetilde{s}_{i}\left(X_{1 i}\right)$. In order to prove this we distinguish two cases. 
(a) $s_{i}\left(X_{1 i}\right)=$ no. We have to show that also $\widetilde{s}_{i}\left(X_{1 i}\right)=$ no. According to the definition of the threshold strategy $\widetilde{s}_{i}$ this means we have to show that

$$
t_{i}\left(s_{i}\right)<p\left(X_{1 i}\right) .
$$

In order to do that, notice that in this case $\lambda_{1}=-1$ according to Lemma 3.3. So,

$$
\begin{aligned}
t_{i}\left(s_{i}\right) & =p\left(H_{R, i}\left(s_{i}\right)\right)+\beta \\
& =2^{R-1}+\sum_{k=1}^{R-1} \lambda_{k} 2^{R-k-1}+\beta \\
& =p\left(X_{1 i}\right)-2^{R-2}+\sum_{k=2}^{R-1} \lambda_{k} 2^{R-k-1}+\beta \\
& \leq p\left(X_{1 i}\right)-2^{R-2}+\sum_{k=2}^{R-1} 2^{R-k-1} \\
& =p\left(X_{1 i}\right)-2^{R-2}+\left(2^{R-2}-2^{R-R}\right) \\
& =p\left(X_{1 i}\right)-2^{R-R}<p\left(X_{1 i}\right) .
\end{aligned}
$$

(b) $s_{i}\left(X_{1 i}\right)=$ yes. In this case we have to show that

$$
t_{i}\left(s_{i}\right) \geq p\left(X_{1 i}\right)
$$

In this case $\lambda_{1}=1$ according to Lemma 3.3 and like in (a) we find that

$$
\begin{aligned}
t_{i}\left(s_{i}\right) & =p\left(X_{1 i}\right)+2^{R-2}+\sum_{k=2}^{R-1} \lambda_{k} 2^{R-k-1}+\beta \\
& \geq p\left(X_{1 i}\right)+2^{R-2}-\sum_{k=2}^{R-1} 2^{R-k-1}-1 \\
& =p\left(X_{1 i}\right)+2^{R-2}-\left(2^{R-2}-2^{R-R}\right)-1 \\
& =p\left(X_{1 i}\right) .
\end{aligned}
$$

This concludes the proof of (2) and (3) for round $r=1$.

(II) Now suppose that (1), (2) and (3) are true in round $r$. We will show that they are also true for $r+1$. First we will show (1). We want to prove this by applying Lemma 2.2 to $a^{r}$ and $b^{r}$. So, we have to show that our situation here satisfies the conditions stated in this lemma. At least it is clear that $a^{r}$ and $b^{r}$ have the same length. So, according to (3) of the induction hypothesis we can indeed apply Lemma 2.2 and find that $A\left(a^{r}\right)=A\left(b^{r}\right), D\left(a^{r}\right)=D\left(b^{r}\right)$ and $W\left(a^{r}\right)=W\left(b^{r}\right)$, which proves (1).

In order to prove (2), suppose that player $i$ is active in round $r+1$ according to $a$. Then player $i$ is certainly also active in round $r$ according to $a$. So, we know from the induction hypothesis that $a^{r}=b^{r}$. 
So, since $\widetilde{s}_{j}=s_{j}$ for all players $j \neq i$, it is then clear that $a_{r+1, j}=b_{r+1, j}$ for all players $j \neq i$. Thus, the only thing left to show is

$$
s_{i}\left(H_{r+1, i}\left(s_{i}\right)\right)=\widetilde{s}_{i}\left(H_{r+1, i}\left(s_{i}\right)\right) .
$$

Again we distinguish two cases.

(a) $s_{i}\left(H_{r+1, i}\left(s_{i}\right)\right)=$ no. We have to show that

$$
t_{i}\left(s_{i}\right)<p\left(H_{r+1, i}\left(s_{i}\right)\right) .
$$

In order to do that notice that, since we assumed that player $i$ is active in round $r+1$ according to $a$, we have $\lambda_{r+1}=-1$ according to Lemma 3.3. So,

$$
\begin{aligned}
t_{i}\left(s_{i}\right) & =p\left(H_{R, i}\left(s_{i}\right)\right)+\beta \\
& =2^{R-1}+\sum_{k=1}^{R-1} \lambda_{k} 2^{R-k-1}+\beta \\
& =p\left(H_{r+1, i}\left(s_{i}\right)\right)+\sum_{k=r+1}^{R-1} \lambda_{k} 2^{R-k-1}+\beta \\
& =p\left(H_{r+1, i}\left(s_{i}\right)\right)-2^{R-r-2}+\sum_{k=r+2}^{R-1} \lambda_{k} 2^{R-k-1}+\beta \\
& \leq p\left(H_{r+1, i}\left(s_{i}\right)\right)-2^{R-r-2}+\sum_{k=r+2}^{R-1} 2^{R-k-1} \\
& =p\left(H_{r+1, i}\left(s_{i}\right)\right)-2^{R-r-2}+\left(2^{R-r-2}-1\right) \\
& =p\left(H_{r+1, i}\left(s_{i}\right)\right)-1<p\left(H_{r+1, i}\left(s_{i}\right)\right) .
\end{aligned}
$$

The other case again goes along the same lines of reasoning.

In order to show (3), notice that we only need to prove the statement in case $k=r+1$. If on the one hand player $i$ is a member of $A\left(a^{k-1}\right)=A\left(a^{r}\right)$, the conclusion immediately follows from the previous one. If on the other hand player $i$ is not a member of $A\left(a^{k-1}\right)=A\left(a^{r}\right)$, the statement follows from the fact that $\widetilde{s}_{j}=s_{j}$ for all players $j \neq i$.

(4) This conclusion immediately follows from the observation that information sets only depend on the number of positive votes, which is always the same in both histories according to (1), and the own actions, which are also always the same since $\widetilde{s}_{j}=s_{j}$ for all players $j \neq i$.

(5) Observe that the payoff is a function of the information sets reached in round $R$ and decisions of active players made in the last round. And all these are the same.

Corollary 3.6 Any strategy $s_{i}$ of a player $i$ can be represented by an equivalent threshold strategy $\widetilde{s}_{i}$. 
Since in the remainder of the paper we only focus on issues involving Nash equilibrium and payoff dominance, this corollary enables us to safely restrict our attention to threshold strategies only. Thus from now on we will no longer talk about strategies in general, but only about threshold strategies, and denote them just by referring to the threshold.

\subsection{Playing the game with threshold strategies}

Next we show the main results of this paper, i.e. that the winner of the object to be sold is a player with the maximum threshold, the price equals the second-highest threshold and consequently that truth-telling is a weakly dominant strategy. In this context we need a couple of notational conventions.

Let $t=\left(t_{i}\right)_{i \in N}$ be a profile of thresholds. This profile will remain fixed during the next few statements. Let us denote the terminal node where the game ends according to this profile by $\tau^{*}$. Let $p(r)=p\left(\tau^{* r-1}\right)$ be the price in round $r$ for this realization of the game.

Definition 3.7 The maximum threshold $t_{\max }$ is defined to be the number $\max _{i \in N} t_{i}$.

Definition 3.8 Let $k$ be a player with $t_{k}=t_{\max }$. The second-highest threshold $t_{\text {sec }}$ is defined to be the number $\max _{i \in N /\{k\}} t_{i}$.

Before we state the next theorem, we need one more notation. First write $I_{00}=\left[0,2^{R}\right)$. Next, for a round $r$ and an integer $d \in\left\{0, \ldots, 2^{r}-1\right\}$, we define

$$
I_{d, r}=\left[d 2^{R-r},(d+1) 2^{R-r}\right) .
$$

Notice that this yields a partition of the interval $I_{00}$ in $2^{r}$ equal-sized intervals.

Theorem 3.9 Let $t=\left(t_{i}\right)_{i \in N}$ be a profile of thresholds played in the bisection auction. The winner of the game is necessarily a player whose threshold equals the maximum threshold.

Proof. According to the definition the set of candidate winner(s) of the game is

$$
W I N\left(\tau^{*}\right)= \begin{cases}W\left(\tau^{*}\right) & \text { if } W\left(\tau^{*}\right) \neq \emptyset \\ A\left(\tau^{*}\right) & \text { otherwise }\end{cases}
$$

Case $1 W\left(\tau^{*}\right) \neq \emptyset=\{k\}$

Take a look at round $r$ in which player $k$ became the winner. He could become the winner only if $a_{r k}=y e s$ and $a_{r j}=n o$ for all $j \neq k$. According to the definition of a threshold strategy it happens only if $p(r)>t_{j}$ for all $j \neq k$ and $p(r) \leq t_{k}$. So, $t_{j}<p(r) \leq t_{k}$. From here it follows that $t_{j}<t_{k}$ for all $j \neq k$. Thus the winner of the game is a player with the highest threshold. 
Case $2 W\left(\tau^{*}\right)=\emptyset$

Let $d(r)$ be the unique index for which $t_{\max }$ is an element of $I_{d(r), r}$. Furthermore, let $j$ be a player with $t_{j}=t_{\max }$ and let $k$ be a player that is a member of $W I N\left(\tau^{*}\right)=A\left(\tau^{*}\right)$. We will show that for all $r \in\{0, \ldots, R\}, t_{k}$ is an element of $I_{d(r), r}$, and consequently $p(r+1)=d(r) 2^{R-r}+2^{R-r-1}$. We will prove this by induction.

For $r=1$. Trivial.

So, assume that $t_{k}$ is an element of $I_{d(r), r}$, and consequently $p(r+1)=d(r) 2^{R-r}+2^{R-r-1}$. We will show this statement for $r+1$. We will consider two cases.

(A) If $\tau_{k, r+1}^{*}=y e s$. Then $p(r+1) \leq t_{k}$ and we know that $t_{k}$ is an element of the interval

$$
I_{2 d(r)+1, r+1}=\left[(2 d(r)+1) 2^{R-r-1},(2 d(r)+2) 2^{R-r-1}\right) .
$$

Moreover,

$$
p(r+1) \leq t_{k} \leq t_{\max }=t_{j}
$$

which implies that $I_{d(r+1), r+1}=I_{2 d(r)+1, r+1}$. Moreover, from Lemma 3.3 we know that, since $k$ is always active, the price in the next round must be the middle of this interval. Hence, $p(r+2)=$ $d(r+1) 2^{R-r-1}+2^{R-r-2}$.

(B) If $\tau_{k, r+1}^{*}=n o$. Then $p(r+1)>t_{k}$ and we know that $t_{k}$ is an element of the interval

$$
I_{2 d(r), r+1}=\left[2 d(r) 2^{R-r-1},(2 d(r)+1) 2^{R-r-1}\right) .
$$

We will show that $\tau_{j, r+1}^{*}=n o$ as well. Suppose $\tau_{j, r+1}^{*}=y e s$. Then either $j$ is the only player who said yes in this round, or there are at least two players that say yes. However, in the first case player $j$ would be the unique winner and that would contradict the fact $W\left(\tau^{*}\right)$ is the empty set. In the second case player $k$ would become a dropout, which would contradict the fact that he is a member of $A\left(\tau^{*}\right)$.

So, $\tau_{j, r+1}^{*}=n o$ as well which means that

$$
p(r+1)>t_{j}
$$

which in its turn implies that $I_{d(r+1), r+1}=I_{2 d(r), r+1}$. Moreover, from Lemma 3.3 we know that, since $k$ is always active, the price in the next round must be the middle of this interval. Hence, $p(r+2)=$ $d(r+1) 2^{R-r-1}+2^{R-r-2}$. 
Finally, from our statement for the last round $R$ we know that both $t_{k}$ and $t_{j}$ are in $I_{d(R), R}$. However, since the length of this half-open interval equals $2^{R-R}=1$, we know that $\left|t_{j}-t_{k}\right|<1$. Hence, since $t_{j}$ and $t_{k}$ are integers we have $t_{j}=t_{k}$.

Lemma 3.10 Let $t=\left(t_{i}\right)_{i \in N}$ be a profile of thresholds played in the bisection auction. A player with the second highest threshold remains active till the end of the game.

Proof. Let player $j$ be a player with the second highest threshold. We have to show that $j \in A\left(\tau^{r}\right)$ for all $0 \leq r \leq R$. We show this inductively for each round $r$. Of course for $r=0$ this is automatically true since in the first round all players are active.

Suppose it is true for some $r$. We have to show that it is true for $r+1$. There are three situations concerning the winner set that could possibly occur. We will discuss these cases separately.

(A) $W\left(\tau^{r+1}\right)=\emptyset$. It means that $\left|P\left(\tau^{r+1}\right)\right| \neq 1$. Suppose $\left|P\left(\tau^{r+1}\right)\right|=0$. Then $A\left(\tau^{r+1}\right)=A\left(\tau^{r}\right)$ - all active players remained active, thus player $j$ too. Suppose $\left|P\left(\tau^{r+1}\right)\right|>1$. Then every player with the highest threshold, as well as any player with the second highest threshold, decides yes. This obviously includes player $j$. And since in this case all players from $A\left(\tau^{r}\right)$ with positive decision remain active, player $j$ will remain active too.

(B) $\quad W\left(\tau^{r}\right)=\emptyset$ and $W\left(\tau^{r+1}\right) \neq \emptyset$. It means that $\left|P\left(\tau^{r+1}\right)\right|=1$. So the only positive decision is the decision of the, in this case unique, player with the highest threshold, and he becomes the winner in this round. The set of active players is $A\left(\tau^{r+1}\right)=A\left(\tau^{r}\right) / W\left(\tau^{r}\right)$. Thus player $j$ remains active.

(C) $W\left(\tau^{r}\right) \neq \emptyset$. For this case it holds that $\left|P\left(\tau^{r+1}\right)\right| \geq 1$. Suppose $\left|P\left(\tau^{r+1}\right)\right|=1$. Then all players from $A\left(\tau^{r}\right)$ have made a negative decision and remain active, thus player $j$ too. If $\left|P\left(\tau^{r+1}\right)\right|>1$, then at least one player from $A\left(\tau^{r}\right)$ has made a positive decision and remains active. This surely includes player $j$ because he has the highest threshold among the players that are still active.

Thus, whatever situation occurred, player $j$ remains active after round $r+1$.

Lemma 3.11 Let $t=\left(t_{i}\right)_{i \in N}$ be a profile of thresholds played in the bisection auction. The price of the terminal node $\tau^{*}$ is equal to the threshold of any player that is active at the end of the game.

Proof. Consider a player from $A\left(\tau^{R}\right)$, say $i$. First of all we will show that $\left|t_{i}-p(R)\right| \leq 1$. We prove this inductively by showing that $\left|t_{i}-p(r)\right| \leq 2^{R-r}$ for each round $r$.

(A) For $r=1$. The price of the first round is equal to $2^{R-1}$. Since $t_{i} \in\left[0,2^{R}\right)$, it is obvious that $\left|t_{i}-p(1)\right| \leq 2^{R-1}$.

(B) Suppose it is true for some $r$, so $\left|t_{i}-p(r)\right| \leq 2^{R-r}$. To show that it's true for $r+1$. We will consider two possible cases. 
(a) $p(r) \leq t_{i}$. Together with the assumption of the induction hypothesis it gives us $p(r) \leq t_{i} \leq$ $p(r)+2^{R-r}$. Moreover, in this case player $i$ has made a positive decision in round $r$. Since he is active in all rounds (and in round $r+1$, too) the price went up and became in round $r+1$ equal to $p(r)+2^{R-r-1}$. From here it automatically follows that $\left|t_{i}-p(r+1)\right| \leq 2^{R-r-1}$.

(b) $p(r)>t_{i}$. For this situation we show in the same way that $p(r)-2^{R-r} \leq t_{i} \leq p(r)$ and $p(r)=p(r+1)+2^{R-r-1}$ from which it automatically follows that $\left|t_{i}-p(r+1)\right| \leq 2^{R-r-1}$.

Thus, $\left|t_{i}-p(R)\right| \leq 1$ for any player who is active at the end of the game. Using this result we will now show that $p\left(\tau^{*}\right)=t_{j}$. In order to do that, notice that the price of the object in the terminal node $\tau^{*}$ is given by

$$
p\left(\tau^{*}\right)= \begin{cases}p(R)-1 & \text { if }\left|P\left(\tau^{*}\right)\right| \leq 1 \\ p(R) & \text { otherwise }\end{cases}
$$

Case 1. $\quad p\left(\tau^{*}\right)=p(R)-1$ and so $\left|P\left(\tau^{*}\right)\right| \leq 1$.

This last inequality implies that player $j$ said no in the last round. That could have happened only if $p(R)>t_{j}$. Together with $\left|t_{j}-p(R)\right| \leq 1$ and $t_{j}, p(R)$ integer, it gives us $p(R)-t_{j}=1$ and consequently $p\left(\tau^{*}\right)=t_{j}$.

Case 2. $\quad p\left(\tau^{*}\right)=p(R)$ and so $|P(\tau)|>1$.

This last inequality implies that player $j$ said yes in the last round. That could have happened only if $p(R) \leq t_{j}$. Let's consider the last round $q$ in which player $j$ has said no (and the price went down last). For player $j$ it holds that $t_{j}<p(q)$. According to Lemma 3.3, since $\lambda(q)=-1$, the price of round $q+1$ was equal to $p(q)-2^{R-q-1}$. Since for any round $r>q$, player $j$ has made the decision yes, $\lambda(r)=1$ and according to Lemma 3.3 the price of the last round is given by $p(R)=p(q+1)+\sum_{k=q+1}^{R-1} 2^{R-k-1}=$ $p(q+1)+2^{R-(q+1)}-1$. From that it follows that $p(q)-p(R)=1$. Thus we have $t_{j}<p(q)$ and $p(q)=p(R)+1$, which means that $t_{j}<p(R)+1$. Together with $p(R) \leq t_{j}$ and $t_{j}, p(R)$ integer, we find $p(R)=t_{j}$ and consequently $p\left(\tau^{*}\right)=t_{j}$.

Theorem 3.12 Let $t=\left(t_{i}\right)_{i \in N}$ be a profile of thresholds played in the bisection auction. The price the winner of the game pays is equal to the second highest threshold.

Proof. According to Lemma 3.11 the price of terminal node $\tau^{*}$ is equal to the threshold of a player who is active at the end of the game. In Lemma 3.10 it has been proven that a player with the second highest threshold is active at the end of the game. Thus, the price of terminal node $\tau^{*}$ is equal to the threshold of a player with the second highest threshold and that is exactly what the winner pays. 
Definition 3.13 The truth-telling strategy of player $i$ is the threshold strategy for which $t$ is equal to player $i$ 's valuation $v_{i}$.

Theorem 3.14 The truth-telling strategy of player $i$ is a weakly dominant strategy.

Proof. From the previous results, specifically Corollary 3.6 and Theorems 3.9 and 3.12, it follows that the bisection auction is strategically equivalent ${ }^{5}$ to the one-shot Vickrey auction. Thus, also in the bisection auction, telling the truth is a weakly dominant strategy.

A final immediate consequence of this theorem is the following corollary.

Corollary 3.15 The truth-telling strategy profile constitutes a symmetric Nash equilibrium. It is the unique perfect Nash equilibrium.

\section{Some remarks on speed and information requirements}

In the previous section we have shown that the bisection auction is strategically equivalent to the Vickrey auction and hence also to the English auction. But the bisection auction is faster and more efficient than the English auction from a computational point of view. This can for example be seen from the worst case analysis. A natural way of measuring the speed of an algorithm is to count the number of operations that it takes until the algorithm ends, and compare this to the length of the input to the algorithm. The length of the input is measured by the number of bits needed to store it in a computer system. In our case, if there are $n$ bidders competing on the interval $\left[0,2^{R}\right)$ in the auction, the entire information has length at most $n R$. With a minimum increment of 1 , the English auction may take up to $2^{R}$ rounds. The running time of the English auction is thus in the worst case of exponential size relative to the length of the input. The bisection auction needs a number of rounds that is logarithmic in the length of the interval $\left[0,2^{R}\right)$, thus it has a running time that is linear in the length of the encoding. Furthermore, if we want to increase the precision with a factor of one half, in other words set the increment in the English auction equal to $1 / 2$ we may need in the English auction $2^{R}$ more rounds, while the bisection auction only needs one more round.

Let us translate this into practical terms. Assume that the valuations are between 0 and 1024 . The English auction may take up to 1024 rounds. The bisection auction will end after 10 rounds. If we want to guarantee that the English auction ends equally fast, we need to set the minimum bid increment to about 100. Let us assume that the valuation is between 0 and $2^{36}$, (about 68 billion Euros, a range that

\footnotetext{
${ }^{5}$ We can interpret the thresholds from Corollary 3.6 as bids in the Vickrey auction and we consequently get the same results here in the bisection auction as in the classical Vickrey auction.
} 
was reached by the UMTS auctions, see e.g. Jehiel and Molduvanu [6]). Again we need only 36 rounds in our bisection auction, while we need more than 68 billion rounds in the English auction. Even if we set in the English auction the bid increment to about 1 million we might need more than $6800(! !)$ rounds.

At least as important is that the proposed bisection auction is more economical in its demand for information on the valuations of the players and therefore preferred by most real-life bidders. The Vickrey auction format requires all participants to reveal all information they have. In the English auction all bidders except a bidder with the highest valuation reveals full information. The reason is that the other players drop out when the price reaches their valuations. In the bisection auction, only a bidder with the second highest valuation reveals his full information. Other bidders do not have to reveal any information about their valuation after they have dropped out or became the winner.

\section{Conclusions}

We proposed a new auction and analyzed its equilibrium properties. First, we have shown that in the bisection auction, threshold strategies are sufficient from a strategic point of view. Furthermore, we have shown that this auction is incentive compatible, that is truth-telling (choosing your threshold equal to your valuation) is a weakly dominant strategy and the equilibrium that results when everyone tells the truth is efficient in the sense that the player with the highest valuation gets the object.

We have shown that our bisection auction is strategically equivalent to the English and Vickrey auctions. However, the proposed auction is preferred over the Vickrey auction because of its iterative nature and over the English auction because of its speed. Furthermore, participation in the bisection auction is less demanding than in the Vickrey and the English auctions. In the bisection auction less information needs to be revealed to the auctioneer to decide on an allocation and a payment.

\section{References}

[1] Ausubel, L.M. [1997] An efficient ascending-bid auction for multiple objects. Working paper, University of Maryland. http://www.ausubel.com/auction-papers/efficient-ascending-auction-r.pdf

[2] Ausubel, L. and Milgrom, P.R. [2001] Ascending auctions with package bidding. Available from http://www.ausubel.com/auction-papers/package-bidding.pdf

[3] Chwe, M.S-Y. [1989] The discrete bid first auction. Economics Letters, 31, 303-306.

[4] Cramton, P. [1998] Ascending auctions. European Economic Review, 42, 745-756. 
[5] Fujishima, Y., McAdams, D. and Shoham, Y. [1999] Speeding up ascending-bid auctions. In Thomas Dean (Ed.) Proceedings of the Sixteenth International Joint Conference on Artificial Intelligence, pp. $554-563$.

[6] Jehiel, P. and Molduvanu, B. [2001] The European UMTS/IMT-2000 license auctions. Mimeo.

[7] Kagel, J.H., Hartstad, R.M. and Levin, D. [1987] Information impact and allocation rules in auctions with affiliated private values: a laboratory study. Econometrica, 55, 1275-1304.

[8] Klemperer, P.D. [1999] Auction theory: A guide to the literature. Journal of Economic Surveys, 13, 227-286.

[9] Milgrom, P.R. and Weber, R.J. [1982] A theory of auctions and competitive bidding. Econometrica, $50,1089-1122$.

[10] Milgrom, P.R. [1989] Auctions and bidding: A primer. Journal of Economic Perspectives, 3, 3-22.

[11] Myerson, R.B. [1981] Optimal auction design. Mathematics of Operations Research, 6, 58-73.

[12] Nisan, N. and Segal, I. [2001] The communication complexity of efficient allocation problems. Available from http://www.cs.huji.ac.il/ noam/mkts.html

[13] Nisan, N. and Blumrosen, L. [2002] Auctions with severely bounded communication. In Dagstuhl seminar on Electronic Market Design. Available from http://www.cs.huji.ac.il/ noam/mkts.html

[14] Rothkopf, M.H., Tisberg, T.J. and Kahn, E.P. [1990] Why are Vickrey auctions rare? Journal of Political Economy, 98, 94-109.

[15] Rothkopf, M.H. and Harstad, R.M. [1994] On the role of discrete bid levels in oral auctions. European Journal of Operational Research, 74, 572-581.

[16] Vickrey, W. [1961] Counterspeculation, auctions and competitive sealed tenders. Journal of Finance, 16, 8-37. 